\title{
How well does the reproductive tract manage infectious agents?
}

\author{
David F. Albertini ${ }^{1}$
}

Published online: 1 October 2016

(C) Springer Science+Business Media New York 2016

Learning to accommodate resident flora via an immunomodulatory détente is fast becoming a fundamental principle in the development and function of organ systems. And the ramifications of coping with host-pathogen interactions in the reproductive tract loom large in a world confronting the scourge of STDs superimposed upon our physiological microbiotathose organisms that have taken up residence in the reproductive tract across our lifespans. Given this, and the importance of the immune system in pregnancy recognition, efforts are ongoing to identify the underlying mechanisms of tolerance operative across the spectrum of human development. Teasing apart inherent benefits obtained by our resident microbiota relative to the demands imposed by maintaining term gestation remains a challenging task for scientists and clinicians alike. Further complicating matters are the pregnancy risks associated with infections in late stage gestations.

In a recent paper, Modi and colleagues draw attention, albeit in an animal model, to the pathogenic mechanisms elicited by bacterial invaders that compromise pregnancy (1). What makes this paper stand out is the identification of membrane vesicles derived from Group B Streptococcus that bear a complement of pore-making toxins and enzymes capable of disrupting the mechanical integrity of the chorion and amnion, resulting in pre-term birth or fetal demise.

Relevant to this and other areas of infectious disease and the practice of human ARTs are two papers this month that

Capsule That organ systems collaborate with endogenous pathogens to establish and maintain physiological homeostasis is fast becoming a dogma posing serious questions when viewed in the context of diagnosing and treating infertility in humans.

David F. Albertini

dalbertini@thechr.com

1 The Center for Human Reproduction, New York, NY, USA highlight concerns at the other end of the human development spectrum. There is no greater disconcerting observation in the embryology lab than to find signs of contamination in embryo cultures. And transfer of contaminated embryos would be counter indicated in all but the most desperate of circumstances. So it is that Shu and colleagues report one such case in which following zona removal, transferred blastocysts resulted in birth of live young (2). While reports of this nature represent extremely rare occurrences, both the risk the zona pellucida poses as a trap for potentially dangerous viruses and microorganisms and defensive strategies intrinsic to the conceptus or enacted by the uterus are topics deserving of further attention, especially in view of the recent issues raised by the Zika virus epidemic.

What about immune surveillance within the ovarian follicle? This has been well studied in animal models where granulosa cells have been identified to have a robust innate immune system activated in response to infection in more distal components of the reproductive tract (3). Notably then have been many papers attempting to define biomarker residents within the ovarian follicle, ranging from transcriptomes and proteomes of granulosa cells to components of follicular fluid that might in some way be indicative or prognostic for oocyte or embryo quality. But with respect to follicular content and diseases like endometriosis, little work has been done until now. The paper by Singh and colleagues extends many previous studies aiming to identify markers of oocyte and embryo quality in women displaying endometriosis (4).

As highly evolved as the repertoire of agents used by the acquired immune system are throughout the reproductive tract, we remain uneducated in the area of how innate immunity negotiates a truce with the various cell types distributed throughout the ovary, fallopian tubes, uterus, and vagina to assure our fertility status. And this expanding area of research begs for a greater investment in funding if we are ever able to 
reconcile the reliance of the reproductive tract in males and females on the microbiota that both facilitate and compromise our fertility.

\section{References}

1. Surve MV, Anil A, Kamath KG, Bhutda S, Sthanam LK, Pradhan A, et al. Membrane vesicles of group b streptococcus disrupt fetomaternal barrier leading to preterm birth. PLoS Pathog. 2016;12(9): e1005816.
2. Shu Y, Prokai D, Berga S, Taylor R, Johnston-MacAnanny E. Transfer of IVF-contaminated blastocysts with removal of the zona pellucida resulted in live births. J Assist Reprod Genet. 2016. doi:10.1007/s10815-016-0776-3.

3. Price JC, Sheldon IM. Granulosa cells from emerged antral follicles of the bovine ovary initiate inflammation in response to bacterial pathogen-associated molecular patterns via Toll-like receptor pathways. Biol Reprod. 2013;89(5):119.

4. Singh AK, Dutta M, Chattopadhyay R, Chakravarty B, Chaudhury $\mathrm{K}$. Intrafollicular interleukin-8, interleukin-12, and adrenomedullin are the promising prognostic markers of oocyte and embryo quality in women with endometriosis. J Assist Reprod Genet. 2016. doi:10.1007/s10815-016-0782-5. 